\title{
Behavioral and electrophysiological measures of click audibility compared in the cat
}

FRAHCIS B. COLAVITA

UNIVERSITY OF PITTSBURGH

Cats were trained to make an avoidance response to clicks in the presence of different levels of white masking noise. The audibility of the clicks at different masking intensities could be determined from the percentage of CRs made by the animals. Click-evoked responses were also recorded from the left inferior colliculus of each cat in the presence of several intensities of masking noise. The results showed that the noise level that just obscured the click-evoked response was quite close in intensity to the noise level that produced threshold click detection in the behavioral situation. While both behavioral and electrophysiological methods yielded similar measures of auditory sensitivity, the electrophysiological measure was obtained in a fraction of the time required to obtain the behavioral measure.

With few exceptions, prolonged behavioral training and testing has in the past been the only method by which auditory threshold data could be obtained from experimental animals. Exceptions include studies by Hind and Schuknecht (1954), Sutton and Schuknecht (1954), and Kimura, Schuknecht, and Sutton (1956). Hind and Schuknecht examined the relation between behavioral audiograms and evoked cortical potentials in the cat; the sound intensity at different frequencies required to elicit strychnine potentials from the cortex of anesthetized cats was compared with the behavioral audiogram for the same frequencies. It was felt by these investigators that if cortical potentials proved to be a satisfactory measure of auditory function, the relatively long periods of time required for behavioral training could be eliminated.

The results of the Hind and Schuknecht investigation indicated that the two measures of threshold differed by a mean value of only $3 \mathrm{~dB}$ over 65 comparison points. The standard deviation for the threshold differences was $17.4 \mathrm{~dB}$. The authors concluded that the cortical response is a useful indicator of cochlear function, but the relatively high degree of variability associated with cortical potentials precludes using the cortical response in place of behavioral measures of auditory sensitivity. Sutton and Schuknecht (1954) and Kimura, Schuknecht, and Sutton (1954) used a refinement of the method described by Hind and Schuknecht to study hearing losses produced by surgical lesions made in the cochleas of cats.

A more recent comparison of cortical potentials and behavioral thresholds was accomplished by using computer averaging techniques in order to effectively stablize the cortical responses (Rothenberg \& Davis, 1967). In this study it was noted that cortical evoked potentials can provide a close approximation to the behaviorally determined hearing threshold of the chinchilla. Rothenberg and Davis required less than an hour to collect data for an 8-frequency audiogram using the electrophysiological method, whereas obtaining similar sensitivity measures behaviorally would have taken over a week.

Rothenberg and Davis found that tone pip-evoked responses recorded from scalp electrodes in anesthetized chinchillas yielded a "threshold" curve quite similar to one previously determined behaviorally by Miller (1964) for the chinchilla. Rothenberg and Davis suggest that the averaging of evoked responses may provide a fast method of assessing the auditory sensitivity of other species of laboratory animals in addition to the chinchilla.

The present experiment also involved a comparison of auditory thresholds as determined both by behavioral methods and by the averaging of evoked potentials, but there are a number of procedural differences between this study and that of Rothenberg and Davis. First of all, cats rather than chinchillas were used as Ss. Second, both the behavioral and the electrophysiological data were obtained from the same animals. Third, the electrical recordings were made from the inferior colliculus with the animals fully conscious, rather than from the scalp with the animals anesthetized. Fourth, the animals were required to attend to a suprathreshold stimulus of constant intensity in the presence of different levels of masking noise, rather than to a threshold stimulus in the absence of masking noise. Finally, clicks, rather than tone pips, were used as the test stimuli.

\section{METHOO}

The Ss were three adult cats with no obvious hearing losses. For two of the animals, the behavioral thresholds were determined before the electrophysiological ones, while this sequence was reversed for the third animal.

\section{Behavioral Data}

The animals were trained in a double grill box of dimensions $40 \times 18 \times 14$ in. to cross from one side to the other at the onset of a 10 sec train of clicks at a repetition rate of 4 clicks per sec. Fallure to cross from one compartment to the other within $10 \mathrm{sec}$ 
resulted in the animal receiving shock through the bars of the box until an escape response was made. Crossing responses made in the absence of the clicks (spontaneous responses) were also punished with shock. By the later stages of training the animals had stopped making spontaneous responses. The clicks were produced by delivering a $2.5 \mathrm{~V}$ sawtooth pulse to a 4 in. speaker located $6.5 \mathrm{ft}$ from the center of the double grill box. The intertrial intervals ranged from $30 \mathrm{sec}$ to $2 \mathrm{~min}$.

All animals received 10 trials a day until they reached a criterion of three successive days at $90 \%$ CRs or better. Each cat required around 12 days of training to achieve this criterion. Next there followed a number of test days on which white noise at different intensities was continuously present during an experimental session, and the animal had to detect the clicks against this noise background. Failures to respond in the presence of the clicks were punished with shock. White noise intensity was measured with a General Radio Type 1565-A sound level meter (C scale). Between 20 and 40 avoidance trials were run at each white noise intensity, the variable of interest at each naise level being the percentage of trials on which the cats detected the clicks.

\section{Electrophysiological Data}

Each cat was prepared in an aseptic operation with a chronic bipolar electrode located in the left inferior colliculus. The electrodes were constructed by twisting together two pieces of .0067 in. stainless steel wire, insulated with Teflon except for $1 \mathrm{~mm}$ at the tip. An effort was made to place the electrode tip in the apex of the colliculus. The electrode wires were attached to a connector plug that was permanently fixed to Ss head with acrylic cement. A ground lead was attached to a stainless steel screw placed in the skull just anterior to the bregma.

Following recovery from surgery, electrical recordings could be made from the inferior colliculus of the awake animal in the same double grill box used for behavioral training. Unfortunately, behavioral and electrophysiological detection measures could not be obtained simultaneously because the shock punishment used during avoidance training produced artifacts in the recording system that prevented the measurement of evoked responses. Evoked responses were recorded in the double grill box by using a flexible overhead cable that attached to the plug atop Ss head. The animals were exposed to click trains in the presence of white noise of various intensities, just as in the behavioral situation, although no shock punishment was associated with this phase of the experiment. The click-evoked responses of the inferior colliculus were amplified by two Tektronix Type 122 preamplifiers, and averaged by a Mnemotron Model $400 \mathrm{~B}$ computer of average transients (CAT).

Forty click-evoked responses were averaged at each white noise intensity. An averaging of the elec-
Table 1. Percentage of Trials

at Different White Noise Levels on which Clicks Were Detected.

Cats

\begin{tabular}{|c|c|c|c|}
\hline $\begin{array}{c}\text { Masking Intensity } \\
\text { in } \mathrm{dB}\end{array}$ & $\begin{array}{l}\mathrm{C}-2 \\
\% \mathrm{CRs}\end{array}$ & $\begin{array}{l}\mathrm{C}-4 \\
\% \mathrm{CRs}\end{array}$ & \\
\hline 60 & 100 & 100 & 100 \\
\hline 70 & 100 & 100 & 100 \\
\hline 75 & 90 & 60 & 80 \\
\hline 76 & 50 & 20 & 60 \\
\hline 77 & 0 & 0 & 10 \\
\hline 78 & 0 & 0 & 0 \\
\hline
\end{tabular}

trical responses to 40 clicks was chosen since on each trial in the behavioral situation the animals had been required to attend to 4 clicks per sec for $10 \mathrm{sec}$. The presence or absence of a click-evoked response at each white noise level was determined visually from the 40 averaged events stored in the CAT.

\section{Behavioral Data}

\section{AESULTS}

The percentage of trials on which the animals detected the clicks at several representative white noise intensities is shown in Table 1 . Click detection can be seen to remain quite good up to a masking noise intensity of $75 \mathrm{~dB}$ SPL. The least sensitive of the animals could still detect the clicks on $60 \%$ of the trials at this noise level. As the masking noise intensity increased past $75 \mathrm{~dB}$, detection fell off sharply, reaching the zero point at $77 \mathrm{~dB}$ for two animals, and at $78 \mathrm{~dB}$ for the third.

It is reasonable to assume that the percentage of avoidance responses at each white noise intensity is a good measure of click detectability, as all failures to make the crossing response in the presence of the clicks were punished with shock. If threshold for click detection is defined in the classical manner, that is, as the point where the animal was correct $50 \%$ of the time, then the threshold for click detection occurred at a masking intensity of between 75 and 76 dB SPL.

\section{Electrophysiological Data}

Tracings of the evoked response averaged from 40 clicks for each of the three animals at representative noise levels are shown in Fig. 1. It can be seen that introduction of white noise even at intensities that had no apparent effect upon the behavioral detection of the clicks brought about significant decrements in evoked response size. When the masking noise intensity 


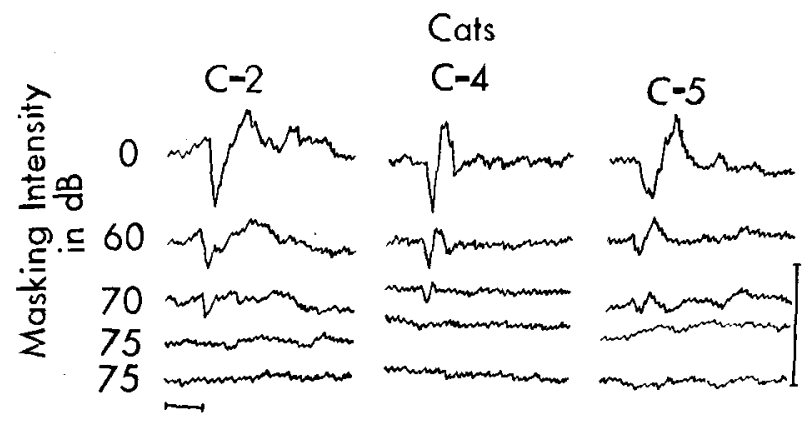

Fig. 1. Tracings of averaged click-evoked responses at different masking intensities. Bottom trace, obtained when no clicks were presented, is included for comparison purposes. Standards represent 10 msec and $200 \mathrm{uV}$.

reached $75 \mathrm{~dB}$ SPL, averaging the electrical activity of the inferior colliculus during 40 click presentations no longer resulted in a visually discernable evoked response. The bottom two traces in Fig. 1 suggest that the wave forms at $75 \mathrm{~dB}$ masking level are indistinguishable whether or not the clicks are actually present.

\section{DISCUSSION}

Considered together, the data in Table 1 and Fig. 1 allow a comparison to be made between a behavioral and an electrophysiological measure of stimulus detectability in the same animal. The averaged evoked response data were collected in increasing steps of $1 \mathrm{~dB}$ starting at $70 \mathrm{~dB}$ masking intensity. When the masking noise intensity reached $75 \mathrm{~dB} S P L$, it was no longer possible to observe an evoked response in any of the cats, although they could still detect the clicks in the behavioral situation. Reductions in evoked response size produced by introducing lesser noise intensities had no counterpart as far as the behavioral measure of detection was concerned.

It should be noted that Rothenberg and Davis averaged a minimum of 64 evoked responses in determining their threshold curves, while only 40 events were averaged in the present situation. Increasing the number of responses averaged would most likely have increased the sensitivity of the electrophysiological measure, although even with the relatively small number of electrical responses averaged in the present experiment a fairly close correspondence was noted between the behavioral and electrophysiological indices of detection threshold. That is, the noise intensity at which the click-evoked response could no Ionger be seen was quite close to the classically defined behavioral detection threshold, and within $3 \mathrm{~dB}$ of the noise level at which behavioral detection fell to zero.

The above data are in accord, then, with the earlier findings of Hind and Schuknecht (1954) and the more recent results of Rothenberg and Davis (1967) that auditory evoked potentials can be useful in quickly assessing the sensitivity of the peripheral auditory mechanism in experimental animals. This now appears to be true not only for detection of near threshold stimuli in a situation free of background noise, but also in situations involving detection of suprathreshold stimuli masked by white noise.

The most meaningful comparison of behavioral and electrophysiological measures of auditory detection would be one in which both measures are obtained simultaneously from the same animal. Work is currently under way to allow such a comparison to be made.

\section{References}

Hind, J. E., \& Schuknecht, H. F. A cortical test of auditory function in experimentally deafened cats. J. Acoust. Soc. Amer. 1954, 26, 89-97.

Kimura, R. S., Schuknecht, H. F., \& Sutton, S. Effects of cochlear lesions on the threshold responses of the auditory cortex in chronic experiments. J. comp. physiol. Psychol., 1956, 49, 96104.

Miller, J. C. Auditory sensitivity of the chinchilla in quiet and in noise. J. Acoust. Soc. Amer., 1964, 36, 2010.

Rothenberg, S., \& Davis, H. Auditory evoked response in chinchilla: Application to animal audiometry. Percept.\& Psychophys., 1967, 2, 443-447.

Sutton, S., \& Schuknecht, H. F. Regional hearing losses from induced cochlear injuries in experimental animals. Annals of Otology, Rhinology \& Laryngology, 1954, 63, 1-27.

(Accepted for publication February 15, 1968.) 